\title{
Disponibilidad de $\mathrm{Ca2}+$, Mg2+ y $\mathrm{K}+$ en función de las propiedades del suelo, zona cafetera central de Colombia
}

\section{Availability of $\mathrm{Ca} 2, \mathrm{Mg} 2$ and $\mathrm{K}$ depending on the properties of the soil, central coffee zone of Colombia}

\author{
Disponibilidade de Ca2 +, Mg2 + e K + em função \\ das propriedades do solo, zona central cafeeira \\ da Colômbia
}

\author{
Luz Adriana Lince Salazar ${ }^{1}$, Nelson Rodríguez Valencia² \& Siavosh Sadeghian Khalajabadi³ \\ ${ }^{1}$ Geóloga, Estudiante de maestría en Desarrollo Sostenible y Medio Ambiente en la Universidad \\ de Manizales, Colombia. ${ }^{2}$ Ingeniero Químico, Doctor en Hidráulica y Medio Ambiente. ${ }^{3}$ Ingeniero \\ Agrónomo, Magister en Ciencias Agrarias énfasis Suelos, Doctor en Ciencias Agropecuarias énfasis \\ Fisiología Vegetal. \\ 1, 2, ${ }^{3}$ Centro Nacional de Investigaciones de Café- CENICAFE. Manizales. Colombia. \\ 1luz.lince@cafedecolombia.com, 2Nelson.Rodriguez@cafedecolombia.com, \\ ${ }^{3}$ Siavosh.Sadeghian@cafedecolombia.com³
}

\section{Resumen}

La nutrición mineral se da a partir de los elementos que se encuentran en forma disponible en la solución del suelo, la cual se abastece principalmente de la fase de cambio iónico. Con el objetivo de determinar la disponibilidad de cationes en la solución del suelo y su relación con las propiedades edáficas, se realizó una investigación con suelos provenientes de cinco unidades cartográficas de la zona cafetera central de Colombia. Por cada unidad cartográfica se seleccionó un lote cafetero, en el que se tomaron muestras de suelo a diferentes profundidades y se analizaron las características físicas, químicas y mineralógicas, incluyendo la concentración de $\mathrm{Ca}^{2+}, \mathrm{Mg}^{2+}$ y $\mathrm{K}^{+}$en la solución. En las cinco unidades el catión predominante fue $\mathrm{Ca}^{2+}$, seguido por $\mathrm{Mg}^{2+}$ y K $\mathrm{K}^{+}$, y las concentraciones de éstos para la fase de cambio y la solución, en la mayoría fueron iguales en los primeros $30 \mathrm{~cm}$ de profundidad y diferentes entre unidades, de las cuales las unidades Catarina, Doscientos y Guamal, presentaron los valores más altos y Quindío y Chinchiná los más bajos, lo cual se relacionó con el material parental ya que las tres primeras provienen de rocas máficas y ultramáficas y las dos últimas de materiales de composición intermedia. Finalmente las concentraciones de $\mathrm{Ca}^{2+}$ en la solución se explicaron desde 36,97 \% hasta 88,11 $\%$ por la fase de cambio, las de $\mathrm{Mg}^{2+}$ desde 32,23 $\%$ hasta $97,30 \%$ y las de $\mathrm{K}^{+}$desde $79,06 \%$ hasta $94,68 \%$, mediante modelos lineales que incluyeron nutrientes del suelo y propiedades como $\mathrm{CIC}$, $\mathrm{pH}$ y contenido de arcillas.

Palabras clave: solución del suelo, fertilidad natural del suelo, mineralogía, café, cationes, material parental. 


\section{Abstract}

Plant nutrition depends on the amount of nutrients in the soil solution coming mainly from the ionic exchange phase. In order to determine the cations availability in the soil solution related to edaphic properties, a study with soils from five cartographic units of the Colombian central coffee region was carried out. Per each unit, a coffee planted plot was selected and soil samples were taken at different depths to measure physical, chemical and mineralogical properties, including the $\mathrm{Ca}^{2+}, \mathrm{Mg}^{2+}$ and $\mathrm{K}^{+}$concentrations in the solution. For all units, the dominant cation was $\mathrm{Ca}^{2+}$ followed by $\mathrm{Mg}^{2+}$ and $\mathrm{K}^{+}$. Their concentrations in the exchange phase and soil solution were similar in the surface $30 \mathrm{~cm}$ but different among soil units. Catarina, 200, and Guamal units exhibited the highest values while Quindio and Chinchina the lowest ones, which was related to the parent material since the first three soils are derived from mafic and ultramafic rocks and the other two from intermediate composite materials. Finally the concentrations of $\mathrm{Ca}^{2+}$ in the soil solution were explained in $36.97 \%$ to $88.11 \%$ by the exchange phase, the $\mathrm{Mg}^{2+}$ and $\mathrm{K}^{+}$concentrations were explained in $32.23 \%$ to $97.30 \%$ and in $79.06 \%$ to $94.68 \%$ respectively, by linear models that included soil nutrients and other properties such as $\mathrm{CIC}, \mathrm{pH}$ and clay content.

Keywords: soil solution, natural fertility of soil, parent material, mineralogy, coffee, cations, parent material.

\section{Resumo}

A nutrição mineral ocorre a partir dos elementos que se encontram de forma disponível na solução do solo, a qual se abastece principalmente da fase de troca iônica. Com o objetivo de determinar a disponibilidade de cátions na solução do solo e a sua relação com as propriedades edáficas, realizou-se uma pesquisa com solos provenientes de cinco unidades cartográficas da zona cafeteira central da Colômbia. Por cada unidade cartográfica se selecionou uma área cafeteira, sendo que amostras de solo, a diferentes profundidades, foram tomadas para analisar as características físicas, químicas e mineralógicas, incluindo a concentração de $\mathrm{Ca} 2+, \mathrm{Mg} 2+$ e $\mathrm{K}+$ na solução. Nas cinco unidades o cátion predominante foi $\mathrm{Ca} 2+$, seguido por $\mathrm{Mg} 2+$ e $\mathrm{K}+$; as concentrações destes para a fase de troca e solução foram, em sua maioria, iguais nos primeiros $30 \mathrm{~cm}$ de profundidade e diferentes entre unidades, sendo que as unidades Catarina, Doscientos e Guamal, apresentaram os valores mais altos, enquanto que Quindio e Chinchiná os valores mais baixos. Isto se relacionou com o material vegetal, uma vez que as três primeiras provêm de rochas máficas e ultramáficas e, as duas últimas, de materiais de composição intermédia. Finalmente, as concentrações de $\mathrm{Ca} 2+$ na solução foram explicadas desde $36,97 \%$ até $88,11 \%$ pela fase de troca, as de $\mathrm{Mg} 2+$ desde $32,33 \%$ até $97,30 \%$ e as de $\mathrm{K}+$ desde $76,06 \%$ até $94,68 \%$, mediante modelos lineares que incluíram nutrimentos do solo e propriedades como CTC, pH e conteúdo de argilas.

Palavras-chave: solução do solo, fertilidade natural do solo, mineralogia, café, cátions, material parental

\section{Introducción}

La nutrición mineral se da a partir de los elementos que se encuentran en forma disponible en la solución del suelo, la cual se abastece principalmente de la fase de intercambio iónico que puede pasar en corto tiempo a la solución durante las diferentes etapas del cultivo (Sadeghian, 2014). En la solución del suelo se encuentran los nutrientes en forma iónica, siendo predominantes $\mathrm{Ca}^{2+}, \mathrm{Mg}^{2+}$ $\mathrm{y} \mathrm{K}^{+}$(Lindsay, 1979). Estos cationes cumplen roles fundamentales en la planta; los dos primeros son constituyentes de órganos estructurales y participan en funciones catalizadoras, el tercero interviene en la osmo-regulación y actividad enzimática (Maathuis, 2009). 
Las plantas toman los nutrientes de la solución del suelo mediante diferentes mecanismos los cuales dependen de factores fisiológicos, atmosféricos y edáficos. Con respecto a estos últimos, tiene importancia el contenido del agua en el suelo y la concentración del nutriente en la solución (Smethurst, 2000), los cuales están relacionados con propiedades como densidad aparente (Wilson et al., 2013; Safadoust et al., 2014; Chen et al., 2014), porosidad total (Tormena et al., 1999), contenido de arcillas (Emamgolizadeh, 2015; Safadoust et al., 2014; Leao et al., 2006), pH (Emamgolizadeh, 2015) y contenido de materia orgánica (Emamgolizadeh, 2015), además de meteorización del material parental (Kim y Kim, 2015; Anda et al., 2015).

Para la región cafetera colombiana se cuenta con estudios de los nutrientes en la solución del suelo, enfocados a la respuesta de aplicación de fertilizantes (Henao, 1995; Henao y Delvaux, 2000; Hincapié \& Henao, 2008), relación con otras propiedades del suelo (Arias et al., 2009; Sadeghian \& Zapata, 2012) y su influencia en la nutrición del café (Henao \& Hernández, 2002; Sadeghian, 2010; Sadeghian, 2012).

Para el cultivo del café, los estudios relacionados con la disponibilidad de $\mathrm{Ca}^{2+}, \mathrm{Mg}^{2+}$ y K $\mathrm{K}^{+}$son relevantes, dada la cantidad removida de estos macronutrientes por la cosecha: $36,9 \mathrm{~kg}$ de potasio, $4,3 \mathrm{~kg}$ de calcio y 2,3 kg de magnesio, por $1000 \mathrm{~kg}$ de café almendra (Sadeghian et al., 2006).

Debido a que los antecedentes son pocos para establecer la relación entre la disponibilidad de cationes y la fase de cambio iónico, se realizó una investigación cuyo objetivo fue determinar la disponibilidad de $\mathrm{K}^{+}, \mathrm{Ca}^{2+}, \mathrm{Mg}^{2+}$ en la solución del suelo en función de las características de la fase de cambio iónico.

\section{Materiales y métodos}

Se emplearon suelos de cinco unidades cartográficas de la zona cafetera central de Colombia
(Tabla 1). Por cada unidad se seleccionó una plantación de café variedad Castillo®, a libre exposición solar en la etapa productiva.

En cada sitio se tomaron tres tipos de muestra y se procuró que la última fertilización se hubiera realizado seis meses antes del muestreo; estos fueron: i) muestras disturbadas, tomadas con palín en cuatro puntos dentro del lote a: $0-5 \mathrm{~cm}$, 5-10 cm, $10-20 \mathrm{~cm}$ y $20-30 \mathrm{~cm}$, de profundidad; ii) muestras sin disturbar, tomadas con un cilindro de acero de $25 \mathrm{~cm}^{3}$ de capacidad, en cuatro puntos dentro del lote a las profundidades mencionadas; iii) muestras disturbadas, conformadas a partir de cuatro submuestras, tomadas con palín en cuatro puntos dentro del lote en las mismas profundidades anotadas.

Se evaluaron las siguientes propiedades para las muestras tipo i: $\mathrm{pH}$ (potenciométrico, relación suelo-agua 1:1), Capacidad de Intercambio CatiónicoCIC (acetato de amonio $1 \mathrm{~N} \mathrm{pH} \mathrm{7,0,} \mathrm{valoración}$ por colorimetría Nessler), Carbono Orgánico-CO (Walkley-Black), $\mathrm{K}^{+}, \mathrm{Ca}^{2+}, \mathrm{Mg}^{2+}$ intercambiable (acetato de amonio $1 \mathrm{~N} \mathrm{pH} \mathrm{7,0,} \mathrm{valoración} \mathrm{por} \mathrm{es-}$ pectrofotometría de absorción atómica-EAA), $P$ (Bray II), Fe, Mn, Zn, Cu (EDTA y EAA), B (agua caliente colorimétrica con azometina- $\mathrm{H}$ ), $\mathrm{S}$ (monofosfato de calcio, valoración turbidimétrica), Al (KCI IM-EAA), Textura (pipeta), Retención de humedad (ollas de presión a 33, 100 y $1500 \mathrm{kPa}$ ), Densidad real (picnómetro); además se determinó la concentración de los cationes en la solución del suelo, mediante el método de la centrífuga (7000 rpm). Con las muestras tipo ii se evaluó: Densidad aparente (cilindro de Coile), y con las muestras tipo iii: Mineralogía de la fracción arena (análisis óptico) y arcilla (difracción de rayos $\mathrm{X}$ ).

Para la extracción de la solución se envasaron $250 \mathrm{~g}$ de suelo (previamente secado al aire) en recipientes plásticos de $80 \mathrm{~cm}^{3}$ con orificios en la parte inferior; se saturó por capilaridad con agua grado analítico durante $48 \mathrm{~h}$, se dejó drenar por $15 \mathrm{~min}$ y se aplicó nuevamente agua en exceso (24 mL), y se drenó por $3 \mathrm{~h}$. Posteriormente, las 
muestras se sometieron a dos ciclos de centrifugado, el primero a $2400 \mathrm{rpm}$ durante $20 \mathrm{~min}$, para retirar el agua de fácil drenaje (agua por encima de la capacidad de campo) y el segundo a $7.000 \mathrm{rpm}$ durante 20 min para extraer el agua en equilibrio. Inmediatamente, el agua de equilibrio se transvasó a tubos falcón de $50 \mathrm{~mL}$, y se cuantificó conductividad eléctrica (conductimétrico) y $\mathrm{pH}$ (potenciométrico). Para la evaluación de $\mathrm{Ca}^{2+}, \mathrm{Mg}^{2+}$ y $\mathrm{K}^{+}$, la muestra contenida en los tubos falcón se dejó en reposo por $24 \mathrm{~h}$, se filtró con un papel de tamaño de poro de $8 \mu \mathrm{m}$ y posteriormente se cuantificó por el método de EAA.
La variación de las características en profundidades y entre unidades, se determinó mediante análisis de varianza al $5 \%$ con el software SAS versión 9.4, en los casos en los cuales se presentó variación se realizó la prueba de Duncan al 5\%. Para establecer la relación entre la concentración de los cationes en la solución y las características de la fase sólida, se realizó un análisis de regresión lineal múltiple con una confianza del $95 \%$ y se corroboró la significancia de los parámetros.

Tabla 1. Unidades de suelo de la zona cafetera de Colombia seleccionadas en la investigación.

\begin{tabular}{|c|c|c|c|c|c|}
\hline \multirow{2}{*}{ Característica } & \multicolumn{5}{|c|}{ Unidad cartográfica } \\
\hline & Catarina & Chinchiná & Doscientos & Guamal & Quindío \\
\hline \multicolumn{6}{|c|}{ Ubicación del muestreo } \\
\hline Departamento & Risaralda & Risaralda & Risaralda & Risaralda & Quindío \\
\hline Municipio & Santuario & Pereira & Marsella & Quinchía & Buenavista \\
\hline Vereda & La María & El Retiro & La Linda & Los Medios & Paraguay \\
\hline Material parental & $\begin{array}{l}\text { Esquisto talco- } \\
\text { so biotítico }\end{array}$ & $\begin{array}{l}\text { Ceniza } \\
\text { volcánica }\end{array}$ & $\begin{array}{l}\text { Basalto hornblén- } \\
\text { dico biotítico }\end{array}$ & $\begin{array}{l}\text { Arenisca } \\
\text { olivínica }\end{array}$ & $\begin{array}{c}\text { Ceniza } \\
\text { volcánica }\end{array}$ \\
\hline Taxonomía & $\begin{array}{c}\text { Typic } \\
\text { Eutrudepts }\end{array}$ & $\begin{array}{c}\text { Typic } \\
\text { Hapludands }\end{array}$ & $\begin{array}{c}\text { Typic } \\
\text { Eutrudepts }\end{array}$ & $\begin{array}{c}\text { Typic } \\
\text { Eutrudepts }\end{array}$ & $\begin{array}{c}\text { Typic } \\
\text { Melanudands }\end{array}$ \\
\hline
\end{tabular}

\section{Resultados y discusión}

En la Tabla 2 se presentan las características físicas, químicas y mineralógicas de los suelos analizados. Las concentraciones de $\mathrm{Ca}^{2+}, \mathrm{Mg}^{2+}$ y K${ }^{+}$en la mayoría de los casos, y para ambas fases, no presentaron diferencia estadística en los primeros $30 \mathrm{~cm}$ del perfil; salvo algunas excepciones, principalmente en los primeros $5 \mathrm{~cm}$ de profundidad (Tablas 3 y 4). Según Smethurst (2000) una mayor concentración de nutrientes en la parte superior de la rizósfera puede deberse a la actividad de ácidos orgánicos, enzima fosfatasa y/o quelantes.

En la solución del suelo, las concentraciones de $\mathrm{Ca}^{2+}$ y $\mathrm{Mg}^{2+}$ en la unidad Guamal, $\mathrm{Mg}^{2+}$ en la unidad Doscientos y los tres cationes en la unidad Catarina tuvieron coeficientes de variación medios (>12\% $\leq 60 \%)$; y los tres cationes en las unidades Chinchiná y Quindío, $\mathrm{Ca}^{2+}$ y $\mathrm{K}^{+}$en la unidad Doscientos y $\mathrm{K}^{+}$en la unidad Guamal presentaron coeficientes de variación altos (> $60 \%$ ). Para la fase de cambio, las concentraciones de $\mathrm{K}^{+}$en la unidad Chinchiná, y los tres cationes en las unidades Catarina, Guamal y Quindío presentaron coeficientes de variación medios; y los tres cationes en la unidad Doscientos, y $\mathrm{Ca}^{2+} \mathrm{y} \mathrm{Mg}^{2+}$ en la unidad Chinchiná presentaron coeficientes de variación altos. Estos coeficientes de variación son similares a los reportados por Sadeghian \& Zapata (2012), Henao \& Hernández (2002) y Lince \& Sadeghian (2012) para suelos de la zona cafetera 
colombiana; donde las dos primeras referencias reportan datos tanto para la fase de cambio como para la solución, y son atribuidos en gran parte a la naturaleza de los materiales y al manejo agronómico de los lotes (Cambardella, 1994; Henao \& Hernández, 2002; Sadeghian, 2010; Safadoust et al., 2014). Lo anterior ratifica la importancia de contemplar las fuentes de variación naturales y antrópicas del suelo, como factor determinante del diseño de muestreo.

En las cinco unidades de suelos, en ambas fases, el catión predominante fue $\mathrm{Ca}^{2+}$, seguido por $\mathrm{Mg}^{2+}$ y $\mathrm{K}^{+}$, lo cual concuerda con lo presentado por varios autores (Anda et al., 2015; Henao \& Hernández, 2002; Sadeghian \& Zapata, 2012). La condición de selectividad entre los cationes es explicada por la abundancia de los materiales inorgánicos en el suelo, por la valencia del ion y por el tamaño del radio de hidratación del ion (Sparks, 2003); para el caso de los cationes de interés, la menor concentración en $\mathrm{K}^{+}$se puede relacionar con una menor retención por los coloides debido a su monovalencia, seguido por el $\mathrm{Mg}^{2+}$, que a pesar de ser divalente presenta un radio de hidratación mayor y por tanto es retenido con menos fuerza que el $\mathrm{Ca}^{2+}$; este último, por tener menor radio de hidratación es retenido con mayor fuerza por los coloides del suelo y es menos complejado, lixiviado y/o precipitado, quedando en mayor concentración para las plantas en comparación con los otros dos cationes.

Las concentraciones de los cationes objeto de estudio fueron estadísticamente diferentes entre unidades. En la solución del suelo las mayores concentraciones de $\mathrm{K}^{+}$se presentaron en la unidad Catarina $\left(0,33 \mathrm{mmol} \cdot \mathrm{L}^{-1}\right)$, las de $\mathrm{Ca}^{2+} \mathrm{y} \mathrm{Mg}^{2+}$ en la unidad Guamal $\left(0,58 \mathrm{mmol} . \mathrm{L}^{-1} ; 0,51 \mathrm{mmol} . \mathrm{L}^{-1}\right.$, respectivamente); y las menores concentraciones para los tres cationes en la unidad Chinchiná $\left(\mathrm{Ca}^{2+}=0,18 \mathrm{mmol} \cdot \mathrm{L}^{-1} ; \mathrm{Mg}^{2+}=0,09 \mathrm{mmol} . \mathrm{L}^{-1} ; \mathrm{y} \mathrm{K}^{+}\right.$ $=0,05 \mathrm{mmol}^{-\mathrm{L}^{-1}}$ ) (Figura 1). Para la fase de cambio los mayores valores de $\mathrm{K}^{+}$se registraron en la unidad Doscientos $\left(0,60 \mathrm{cmol}_{c} \cdot \mathrm{kg}^{-1}\right)$, los de $\mathrm{Ca}^{2+} \mathrm{y}$ $\mathrm{Mg}^{2+}$ en la unidad Guamal $\left(14,00 \mathrm{cmol}_{\mathrm{c}} \cdot \mathrm{kg}^{-1} ; 7,31\right.$ $\mathrm{cmol}_{\mathrm{c}} \cdot \mathrm{kg}^{-1}$, respectivamente); y al igual que en la solución del suelo, los valores menores para los tres cationes se reportaron en la unidad Chinchiná $\left(\mathrm{Ca}^{2+}=1,08 \mathrm{cmol}_{\mathrm{c}} \cdot \mathrm{kg}^{-1} ; \mathrm{Mg}^{2+}=0,26 \mathrm{cmol}_{\mathrm{c}} \cdot \mathrm{kg}^{-1}\right.$; $\mathrm{K}^{+}=0,13 \mathrm{cmol}_{\mathrm{c}} \cdot \mathrm{kg}^{-1}$ ) (Figura 2). Los resultados encontrados concuerdan con Henao (1995) quien reportó para los cationes objeto de estudio mayor contenido en la unidad Doscientos en comparación con la unidad Chinchiná. La menor disponibilidad de bases en la unidad Quindío puede estar relacionada con el porcentaje de partículas tamaño arena (50,99 \%) las cuales según Melo, et al. (2009) facilitan el lixiviado y disminuyen la capacidad de intercambiar cationes; sin embargo esta relación no se da con la unidad Chinchiná, la cual presenta las menores concentraciones de bases y un porcentaje de arenas menor que el de otras tres unidades $(18,56 \%)$.

Se encontró relación lineal entre las concentraciones de los cationes de interés y la composición del material parental de los suelos. Las unidades provenientes de materiales de composición ultramáfica (Guamal) y máfica (Catarina y Doscientos) exhibieron los mayores contenidos de $\mathrm{Ca}^{2+}, \mathrm{Mg}^{2+} \mathrm{y} \mathrm{K}^{+}$, mientras que los provenientes de materiales intermedios (Chinchiná y Quindío) presentaron los menores contenidos. Lo anterior concuerda con Anda et al. (2012) en la que hacen referencia a una relación directa entre la cantidad de minerales máficos meteorizados y la disponibilidad de bases en el suelo y se justifica en que los materiales máficos tienen mayores contenidos de piroxenos, anfíboles y plagioclasas, los cuales para Melo et al. (2009) son la mayor fuente primaria de $\mathrm{Ca}^{2+}, \mathrm{Mg}^{2+}$ y K+ en las reservas del suelo.

Dado lo anterior, las unidades Guamal, Doscientos y Catarina poseen una fertilidad potencial mayor que Chinchiná y Quindío, sin embargo, las cinco son de fertilidad potencial alta, debido a que poseen una reserva de minerales de fácil meteorización, los cuales al degradarse aportan elementos básicos para el crecimiento vegetal, además de formar minerales de arcillas (Tabla 2). 
Tabla 2. Características físicas, químicas y mineralógicas de los suelos analizados.

\begin{tabular}{|c|c|c|c|c|c|}
\hline \multirow{2}{*}{ Característica } & \multicolumn{5}{|c|}{ Unidad cartográfica } \\
\hline & Catarina & Chinchiná & Doscientos & Guamal & Quindío \\
\hline \multicolumn{6}{|c|}{ Características químicas* } \\
\hline $\mathrm{pH}$ & 5,27 & 4,81 & 4,84 & 5,61 & 4,96 \\
\hline $\mathrm{CO}, \%$ & 2,82 & 5,43 & 2,60 & 2,3 & 3,6 \\
\hline $\mathrm{Ca} 2+$, cmolc.kg-1 & 5,27 & 1,08 & 7,06 & 14,00 & 2,05 \\
\hline Mg2+, cmolc.kg-1 & 1,56 & 0,22 & 3,33 & 7,13 & 0,32 \\
\hline $\mathrm{K}+$, cmolc.kg-1 & 0,44 & 0,13 & 0,60 & 0,26 & 0,23 \\
\hline $\mathrm{Al} 3+, \mathrm{cmolc} . \mathrm{kg}-1$ & 0,23 & 1,26 & 1,39 & 0,20 & 0,88 \\
\hline P, mg.kg-1 & 7,69 & 6,88 & 4,13 & 3,19 & 44,94 \\
\hline $\mathrm{S}, \mathrm{mg} \cdot \mathrm{kg}-1$ & 8,81 & 16,88 & 21,54 & 8,21 & 11,73 \\
\hline Mn, mg.kg-1 & 56,63 & 33,63 & 73,13 & 155,06 & 27,50 \\
\hline $\mathrm{Cu}, \mathrm{mg} \cdot \mathrm{kg}-1$ & 4,01 & 1,89 & 5,88 & 10,45 & 3,71 \\
\hline $\mathrm{Fe}, \mathrm{mg} \cdot \mathrm{kg}-1$ & 347,13 & 194,13 & 229,88 & 219,44 & 159,94 \\
\hline Zn, mg.kg-1 & 4,03 & 5,06 & 2,32 & 3,24 & 3,41 \\
\hline $\mathrm{B}, \mathrm{mg} \cdot \mathrm{kg}-1$ & 0,44 & 0,26 & 0,32 & 0,37 & 0,53 \\
\hline CIC, cmolc.kg-1 & 17,19 & 26,00 & 22,81 & 21,63 & 16,13 \\
\hline CICE, cmolc.kg-1 & 7,50 & 2,68 & 12,40 & 21,60 & 3,48 \\
\hline \multicolumn{6}{|c|}{ Características físicas* } \\
\hline Arena, \% & 22,24 & 18,56 & 24,83 & 18,02 & 50,99 \\
\hline Limo, \% & 34,69 & 56,80 & 36,36 & 43,21 & 27,50 \\
\hline Arcilla, \% & 42,85 & 24,64 & 38,81 & 38,77 & 21,51 \\
\hline $\begin{array}{l}\text { Densidad aparente, } \\
\text { g.cm-3 }\end{array}$ & 1,19 & 0,72 & 0,95 & 1,10 & 0,96 \\
\hline Densidad real, g.cm-3 & 2,63 & 2,65 & 2,59 & 2,65 & 2,61 \\
\hline \multicolumn{6}{|c|}{ Mineralogía de arenas** } \\
\hline Mineral dominante & Fel, Anf & Fel, Anf, & Fel, Anf & Fel, Anf & Fel, Anf \\
\hline Mineral subdominante & Prx & Prx & Prx & Prx & Qz \\
\hline \multicolumn{6}{|c|}{ Mineralogía de arcillas ${ }^{\star *}$} \\
\hline Mineral dominante & Mnc, & Mnc & Mnc & Mnc & Mnc \\
\hline Mineral subdominante & $\begin{array}{l}\text { KK, Qz, Cri, } \\
\text { Esm, Ver/Mon }\end{array}$ & Ver/Mon & $\begin{array}{l}\text { KK, Ver/Mon, } \\
\text { Cri, Hal }\end{array}$ & $\mathrm{KK}$ & Ver/Mon \\
\hline
\end{tabular}

* Datos correspondientes al promedio de 16 muestras tomadas de 0 a $30 \mathrm{~cm}$ de profundidad en cuatro puntos del lote.

** Datos correspondientes a la moda de 4 muestras dentro del lote tomadas de 0 a $30 \mathrm{~cm}$ de profundidad.

$\mathrm{Fel}=$ feldespato; $\mathrm{Anf}=$ anfíbol; $\operatorname{Prx}=$ piroxeno; $\mathrm{Qz}=$ cuarzo; $\mathrm{Mnc}=$ material no cristalino (vidrio volcánico, carbonatos, sulfatos y fosfatos), $\mathrm{KK}$ = caolinita; Cri = crisotilo; Esm = esmectita; Ver/Mon: vermiculita y/o montmorillonita; Hal = Haloisita. 
Tabla 3. Concentración de las bases intercambiables en la fase de cambio, por profundidades para las unidades cartográficas.

\begin{tabular}{|c|c|c|c|c|c|c|c|c|c|}
\hline \multirow{2}{*}{ Profundidad } & \multicolumn{3}{|c|}{$\mathrm{Ca}^{2+}$} & \multicolumn{3}{|c|}{$\mathrm{Mg}^{2+}$} & \multicolumn{3}{|c|}{$\mathbf{K}^{+}$} \\
\hline & \multicolumn{2}{|c|}{$\begin{array}{l}\text { Promedio } \\
\text { (cmolc.kg-1) }\end{array}$} & \multirow[t]{2}{*}{$\begin{array}{c}\text { CV } \\
(\%)\end{array}$} & \multicolumn{2}{|c|}{$\begin{array}{l}\text { Promedio } \\
\text { (cmolc.kg-1) }\end{array}$} & \multirow[t]{2}{*}{$\begin{array}{l}\text { CV } \\
(\%)\end{array}$} & \multicolumn{2}{|c|}{$\begin{array}{l}\text { Promedio } \\
\left(\text { cmolc.kg-1) }^{-1}\right.\end{array}$} & \multirow[t]{2}{*}{$\begin{array}{l}\text { CV } \\
(\%)\end{array}$} \\
\hline & & & & :atarina & $=4)$ & & & & \\
\hline $0-5$ & 6,017 & $\mathrm{a}$ & 23,23 & 2,048 & $\mathrm{a}$ & 24,90 & 0,750 & a & 45,31 \\
\hline $5-10$ & 4,933 & $\mathrm{a}$ & 11,54 & 1,457 & $\mathrm{~b}$ & 16,68 & 0,468 & $a b$ & 42,94 \\
\hline $10-20$ & 4,855 & $\mathrm{a}$ & 19,79 & 1,355 & $\mathrm{~b}$ & 20,33 & 0,335 & $b$ & 54,09 \\
\hline $20-30$ & 5,278 & a & 17,16 & 1,370 & $\mathrm{~b}$ & 25,24 & 0,223 & $\mathrm{~b}$ & 61,66 \\
\hline \multicolumn{10}{|c|}{ Unidad Chinchiná $(n=4)$} \\
\hline $0-5$ & 1,488 & a & 63,50 & 0,393 & $\mathrm{a}$ & 64,93 & 0,215 & a & 30,50 \\
\hline $5-10$ & 0,820 & a & 107,99 & 0,183 & a & 81,44 & 0,120 & $b$ & 39,09 \\
\hline $10-20$ & 0,748 & $\mathrm{a}$ & 114,86 & 0,148 & $\mathrm{a}$ & 72,68 & 0,093 & $\mathrm{~b}$ & 34,61 \\
\hline $20-30$ & 1,260 & a & 128,67 & 0,138 & a & 98,90 & 0,085 & $\mathrm{~b}$ & 15,19 \\
\hline \multicolumn{10}{|c|}{ Unidad Doscientos $(n=4)$} \\
\hline $0-5$ & 7,593 & a & 35,19 & 3,683 & a & 39,73 & 0,923 & $\mathrm{a}$ & 76,50 \\
\hline $5-10$ & 6,643 & a & 45,81 & 3,143 & a & 48,00 & 0,618 & a & 85,95 \\
\hline $10-20$ & 6,755 & a & 61,63 & 3,128 & a & 68,57 & 0,460 & a & 100,60 \\
\hline $20-30$ & 7,268 & a & 69,53 & 3,383 & a & 69,44 & 0,418 & a & 106,61 \\
\hline \multicolumn{10}{|c|}{ Unidad Guamal $(n=4)$} \\
\hline $0-5$ & 14,745 & a & 35,61 & 7,105 & $\mathrm{a}$ & 30,85 & 0,438 & a & 33,35 \\
\hline $5-10$ & 12,788 & $a$ & 39,32 & 6,595 & $a$ & 35,54 & 0,263 & $a b$ & 49,71 \\
\hline $10-20$ & 13,558 & $\mathrm{a}$ & 34,85 & 7,200 & a & 33,53 & 0,203 & $b$ & 65,31 \\
\hline $20-30$ & 14,918 & $\mathrm{a}$ & 40,46 & 7,638 & a & 37,41 & 0,148 & $\mathrm{~b}$ & 40,06 \\
\hline \multicolumn{10}{|c|}{ Unidad Quindío ( $n=4)$} \\
\hline $0-5$ & 3,085 & a & 32,87 & 0,570 & a & 35,64 & 0,39 & a & 15,53 \\
\hline $5-10$ & 1,955 & $a b$ & 33,70 & 0,320 & $b$ & 32,78 & 0,25 & $b$ & 26,35 \\
\hline $10-20$ & 1,690 & $a b$ & 45,26 & 0,233 & $b$ & 27,96 & 0,16 & c & 18,24 \\
\hline $20-30$ & 1,473 & $b$ & 81,17 & 0,165 & $b$ & 64,61 & 0,12 & c & 6,80 \\
\hline
\end{tabular}

$\mathrm{CV}=$ coeficiente de variación

Letra diferente indica diferencia entre profundidades 
Tabla 4. Concentración de las bases intercambiables en la solución del suelo, por profundidad para las unidades cartográficas.

\begin{tabular}{|c|c|c|c|c|c|c|c|c|c|}
\hline \multirow{2}{*}{ Profundidad } & \multicolumn{3}{|c|}{$\mathrm{Ca}^{2+}$} & \multicolumn{3}{|c|}{$\mathrm{Mg}^{2+}$} & \multicolumn{3}{|c|}{$\mathbf{K}^{+}$} \\
\hline & \multicolumn{2}{|c|}{$\begin{array}{l}\text { Promedio } \\
\left(\text { mmol.L-1) }^{-1}\right.\end{array}$} & $\begin{array}{l}\text { CV } \\
(\%)\end{array}$ & \multicolumn{2}{|c|}{$\begin{array}{l}\text { Promedio } \\
\left(\mathrm{mmol}^{-1}\right)\end{array}$} & $\begin{array}{l}\text { CV } \\
(\%)\end{array}$ & \multicolumn{2}{|c|}{$\begin{array}{l}\text { Promedio } \\
\left(\mathrm{mmol} . \mathrm{L}^{-1}\right)\end{array}$} & $\begin{array}{l}\text { CV } \\
(\%)\end{array}$ \\
\hline & \multicolumn{9}{|c|}{ Unidad Catarina $(n=4)$} \\
\hline $0-5$ & 0,321 & $\mathrm{a}$ & 50,10 & 0,224 & A & 18,31 & 0,587 & $\mathrm{a}$ & 59,66 \\
\hline $5-10$ & 0,228 & a & 15,39 & 0,134 & B & 20,94 & 0,322 & $a b$ & 52,77 \\
\hline $10-20$ & 0,181 & a & 13,95 & 0,099 & B & 21,52 & 0,263 & $a b$ & 48,62 \\
\hline $20-30$ & 0,235 & a & 37,82 & 0,121 & B & 28,96 & 0,157 & $\mathrm{~b}$ & 106,91 \\
\hline \multicolumn{10}{|c|}{ Unidad Chinchiná $(n=4)$} \\
\hline $0-5$ & 0,319 & a & 42,18 & 0,189 & $A$ & 50,58 & 0,124 & a & 42,77 \\
\hline $5-10$ & 0,241 & $a b$ & 86,06 & 0,107 & $a b$ & 61,86 & 0,045 & $\mathrm{~b}$ & 70,68 \\
\hline $10-20$ & 0,089 & $\mathrm{~b}$ & 80,65 & 0,051 & B & 61,80 & 0,028 & $\mathrm{~b}$ & 124,65 \\
\hline $20-30$ & 0,050 & $\mathrm{~b}$ & 49,67 & 0,029 & B & 28,57 & 0,018 & $\mathrm{~b}$ & 164,13 \\
\hline \multicolumn{10}{|c|}{ Unidad Doscientos $(n=4)$} \\
\hline $0-5$ & 0,783 & $\mathrm{a}$ & 19,49 & 0,471 & A & 27,89 & 0,415 & $\mathrm{a}$ & 111,14 \\
\hline $5-10$ & 0,357 & $\mathrm{~b}$ & 82,35 & 0,241 & A & 81,97 & 0,185 & $\mathrm{a}$ & 168,89 \\
\hline $10-20$ & 0,361 & $\mathrm{~b}$ & 45,92 & 0,265 & A & 33,53 & 0,170 & a & 166,14 \\
\hline $20-30$ & 0,328 & $\mathrm{~b}$ & 69,88 & 0,245 & A & 56,07 & 0,160 & $\mathrm{a}$ & 108,95 \\
\hline \multicolumn{10}{|c|}{ Unidad Guamal $(n=3)$} \\
\hline $0-5$ & 1,018 & a & 29,94 & 0,913 & a & 32,32 & 0,145 & a & 43,39 \\
\hline $5-10$ & 0,616 & $\mathrm{~b}$ & 28,01 & 0,539 & $b$ & 36,57 & 0,093 & $\mathrm{a}$ & 154,50 \\
\hline $10-20$ & 0,351 & $\mathrm{bc}$ & 28,12 & 0,298 & $\mathrm{~b}$ & 33,06 & 0,053 & $\mathrm{a}$ & 180,19 \\
\hline $20-30$ & 0,264 & $\mathrm{c}$ & 41,57 & 0,219 & $\mathrm{~b}$ & 49,17 & 0,000 & $a$ & 0,00 \\
\hline \multicolumn{10}{|c|}{ Unidad Quindío $(n=4)$} \\
\hline $0-5$ & 0,629 & $a$ & 54,60 & 0,263 & a & 63,99 & 0,608 & $a$ & 55,16 \\
\hline $5-10$ & 0,271 & $\mathrm{~b}$ & 76,98 & 0,105 & $\mathrm{~b}$ & 69,08 & 0,218 & $\mathrm{~b}$ & 97,38 \\
\hline $10-20$ & 0,309 & $a b$ & 40,91 & 0,109 & $\mathrm{~b}$ & 38,67 & 0,200 & $\mathrm{~b}$ & 75,16 \\
\hline $20-30$ & 0,202 & $\mathrm{~b}$ & 60,16 & 0,074 & $\mathrm{~b}$ & 55,18 & 0,088 & $\mathrm{~b}$ & 46,81 \\
\hline
\end{tabular}

$\mathrm{CV}=$ coeficiente de variación

Letra diferente indica diferencia entre profundidades 

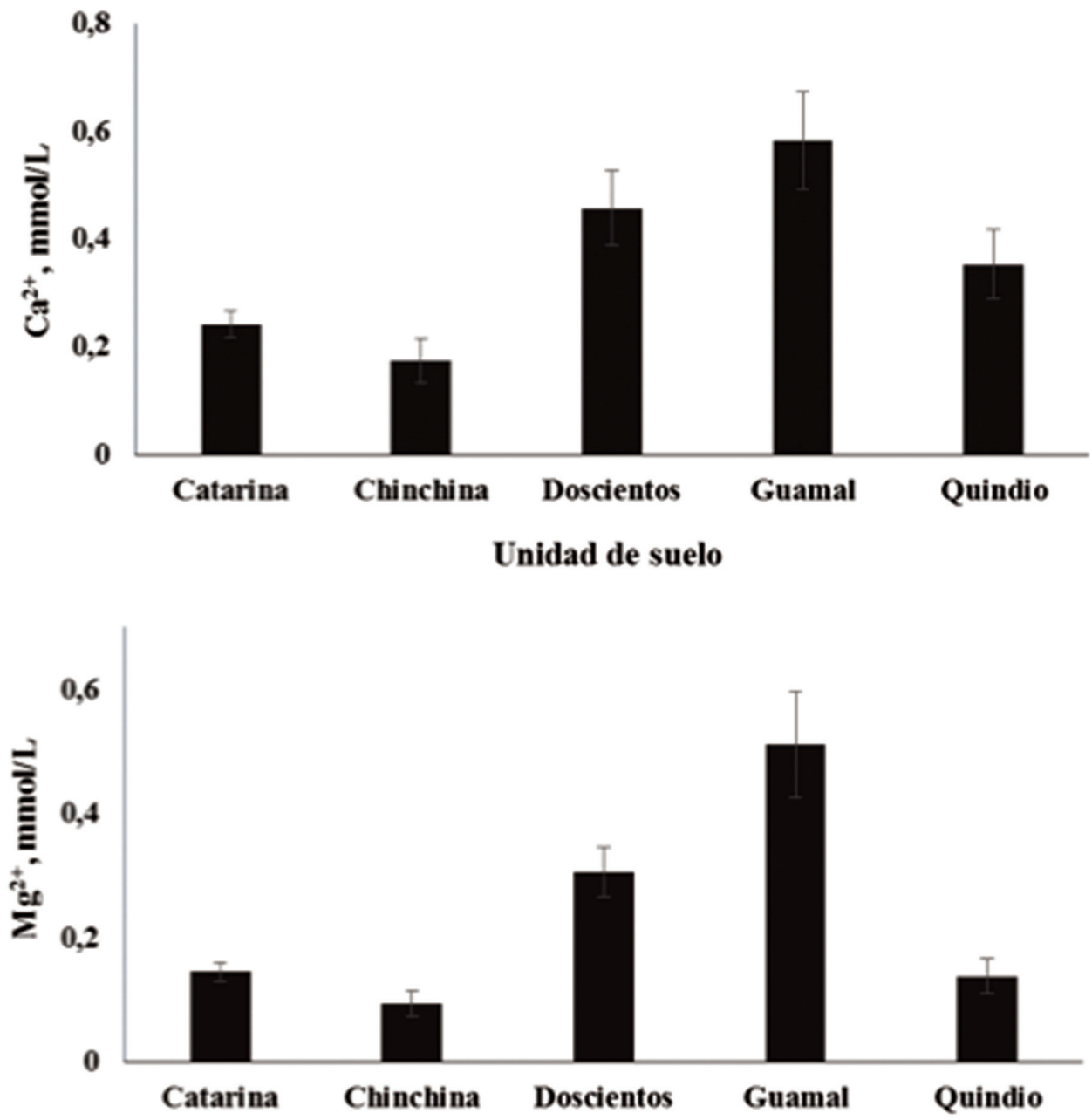

Unidad de suelo

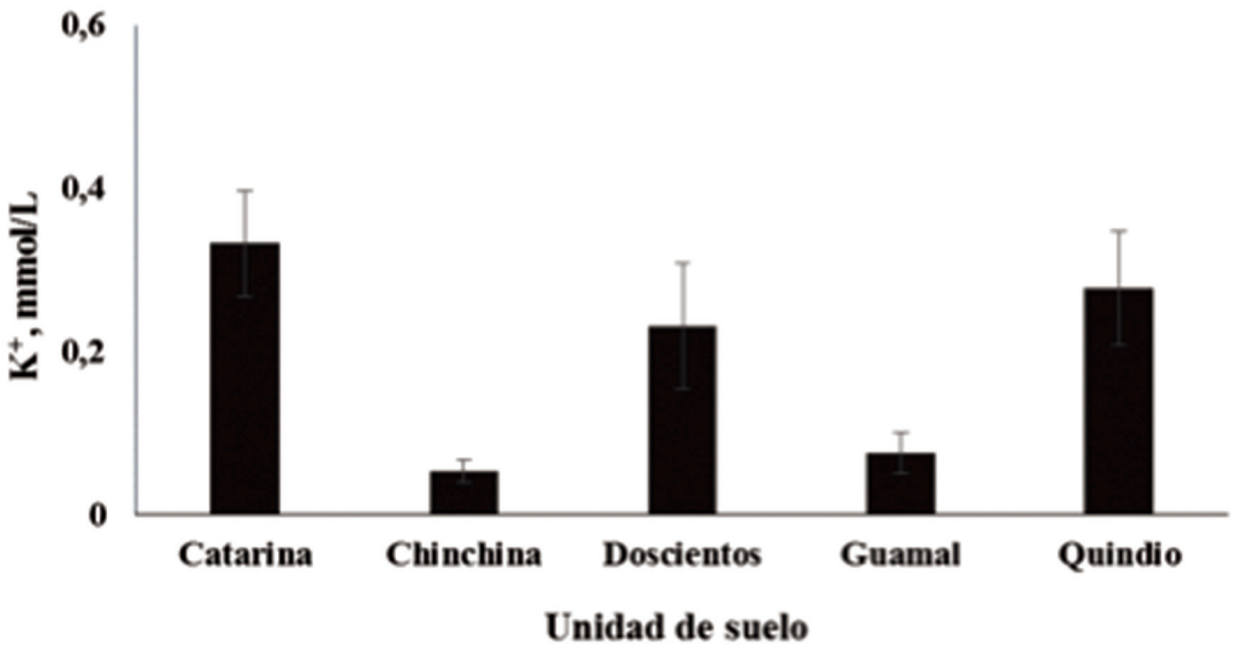

Figura 1. Valores promedio de $\mathrm{Ca}+2, \mathrm{Mg}+2$ y $\mathrm{K}+$ en la solución del suelo, para cinco unidades cartográficas de suelo ( $n=16$ para las unidades Catarina, Chinchiná, Doscientos y Quindío; $n=15$ para la unidad Guamal). La desviación corresponde al error estándar. 

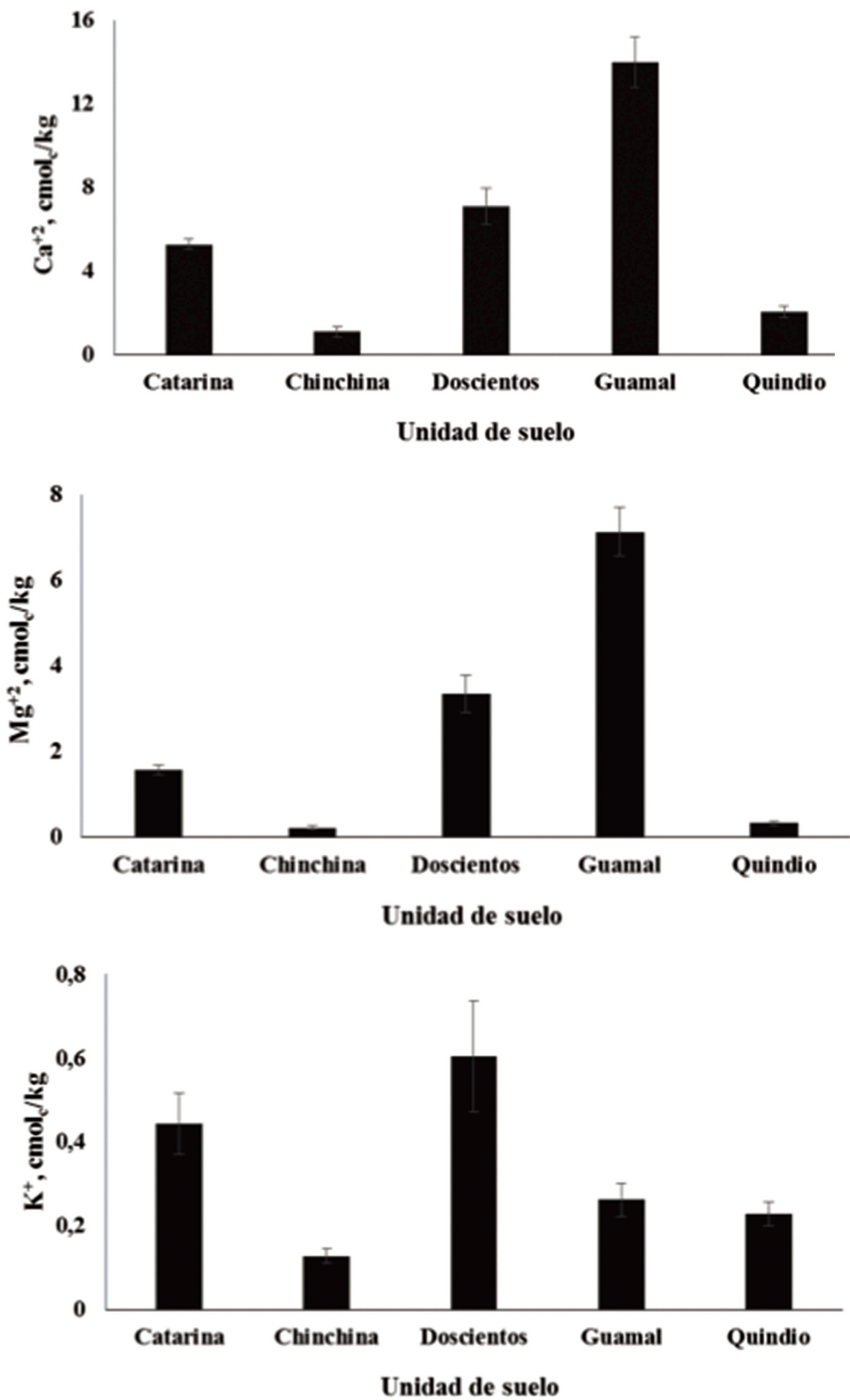

Figura 2. Valores promedio de $\mathrm{Ca}+2, \mathrm{Mg}+2$ y $\mathrm{K}+$ en la solución del suelo, para cinco unidades cartográficas $(n=16)$.

La desviación corresponde al error estándar. 
Las concentraciones de $\mathrm{Ca}^{2+}, \mathrm{Mg}^{2+} \mathrm{y} \mathrm{K}^{+}$en la solución del suelo ${ }_{\mathrm{ss}}$ ), se lograron explicar por la fase de cambio $\left({ }_{\mathrm{fc}}\right)$ mediante modelos lineales generales (se incluyeron todas las unidades), y modelos individuales (para cada unidad), a excepción de la concentración de $\mathrm{Ca}^{2+}$ en la unidad Catarina en la cual las variables edáficas incluidas no pudieron explicar dicha concentración (Tablas 5 y 6).

El $\mathrm{Ca}^{2+}$ ss fue explicado por un modelo general con expresión directa de las variables $\mathrm{K}_{\mathrm{fc}}, \mathrm{P}_{\mathrm{fc}}, \mathrm{Mn}_{\mathrm{fc}}$, el cual difirió de los modelos individuales por un menor $\mathrm{R}^{2}(52,56 \%)$, a excepción de la unidad Doscientos $(36,97 \%)$; además, también difirió por no tener otras variables explicativas como $\mathrm{CIC}$ en Doscientos, $\mathrm{Mg}_{\mathrm{fc}}$ y Ar en Quindío, $\mathrm{S}_{\mathrm{fc}}$ en Guamal. En $\mathrm{Mg}^{2+}$ ss el modelo general fue explicado en relación directa por $\mathrm{K}_{\mathrm{fc}}, \mathrm{Mn}_{\mathrm{fc}}$ y $\mathrm{Cu}_{\mathrm{fc}}$ y en inversa por $\mathrm{Fe}_{\mathrm{fc}}$ y $\mathrm{Na}_{\mathrm{fc}}$ el $\mathrm{R}^{2}$ de éste fue mayor que el de los modelos individuales (74,24\%), a excepción de la unidad Guamal (97,30\%). Finalmente, $\mathrm{K}^{+}{ }_{\text {ss }}$ en el modelo general fue explicado de manera directa por $\mathrm{pH}_{\mathrm{fc}}, \mathrm{K}_{\mathrm{fc}}, \mathrm{P}_{\mathrm{fc}}$ y $\mathrm{Fe}_{\mathrm{fc}}$ e inversa por $\mathrm{Mn}_{\mathrm{fc}}$; en comparación con los modelos por unidad, este incluyó más variables pero el $\mathrm{R}^{2}$ fue similar. Con estos modelos se corroboran las propuestas de otros autores (Anda et al., 2015; Emamgolizadeh et al., 2015) al indicar que hay relación entre la disponibilidad de $\mathrm{Ca}^{2+}, \mathrm{Mg}^{2+}$ y K $\mathrm{K}^{+}$con micronutrientes del suelo, propiedades químicas como $\mathrm{CIC}, \mathrm{pH}$ y contenido de arcillas.

La relación entre la concentración del catión en la solución (intensidad) y la concentración en la fase de cambio (cantidad) en algunos casos se explicó con la presencia del catión en los modelos, como $\mathrm{Mg}^{2+}$ ss en las unidades Catarina, Chinchiná y Quindío y $\mathrm{K}_{\text {ss }}^{+}$en el modelo general y en las unidades $\mathrm{Ca}$ tarina, Chinchiná, Doscientos y Guamal; respecto a este último concuerda con lo reportado por Henao y Hernández (2002) al explicar que existe una relación lineal entre el potasio intercambiable y el potasio en solución, con variaciones en la pendiente de los modelos de cada unidad. En los modelos en los cuales la concentración del catión en solución no se explicó en función del catión en la fase de cambio, como $\mathrm{Ca}^{2+}{ }_{s s}$ en ninguno de los modelos, $\mathrm{Mg}^{2+}{ }_{s s}$ en las unidades Doscientos y Guamal y $\mathrm{K}_{\text {ss }}^{+}$en Quindío, se puede deber a lo que Arias et al. (2009), Melo et al. (2009) y Sparks (2003) identifican como consecuencia de las interacciones entre iones, la fijación por parte de las arcillas y la influencia de la $\mathrm{CIC}$ y el pH.

Tabla 5. Modelos de regresión lineal para las concentraciones de Ca2+, Mg2+ y K+ en la solución del suelo en función de la fase de cambio de las unidades cartográficas.

\begin{tabular}{|c|c|}
\hline Modelo $(n=80)$ & $\mathbf{R}^{2}$ \\
\hline $\mathrm{Ca}^{2+}{ }_{\mathrm{ss}}=0,1541+5,8308^{\star} \mathrm{K}_{\mathrm{fc}}+0,1782^{\star * \star} \mathrm{P}_{\mathrm{fc}}+0,1437^{\star \star *} \mathrm{Mn}_{\mathrm{fc}}$ & 52,56 \\
\hline $\mathrm{Mg}^{2+}{ }_{\mathrm{ss}}=2,6494^{\star}+2,7449^{\star} \mathrm{K}_{\mathrm{fc}}-53,1747^{\star} \mathrm{Na}_{\mathrm{fc}}-0,0125^{\star \star} \mathrm{Fe}_{\mathrm{fc}}+0,0569^{\star \star \star} \mathrm{Mnfc}+0,5451^{\star \star \star} \mathrm{Cu}_{\mathrm{fc}}$ & 74,24 \\
\hline $\mathrm{K}^{+}{ }_{\mathrm{ss}}=-24,2877^{* *}+3,8089^{*} \mathrm{pH}_{\mathrm{fc}}+22,0402^{* * *} \mathrm{~K}_{\mathrm{fc}}+0,1880^{\star * *} \mathrm{P}_{\mathrm{fc}}+0,0194^{* *} \mathrm{Fefc}-0,0269^{\star} \mathrm{Mn}_{\mathrm{fc}}$ & 80,27 \\
\hline
\end{tabular}

ss = solución del suelo; ${ }_{\mathrm{fc}}=$ fase de cambio; ${ }^{*}=$ valor $p<0,05 \geq 0,01 ;{ }^{* *}=$ valor $p<0,01 \geq 0,001 ;{ }^{* *}=$ valor $p<0,001$ 
Tabla 6. Modelos de regresión lineal para las concentraciones de Ca2+, Mg2+ y K+ en la solución del suelo en función de la fase de cambio, para cada unidad cartográfica

\begin{tabular}{|c|c|c|}
\hline Unidad & Modelo $(n=16)$ & $\mathbf{R}^{2}$ \\
\hline Chinchiná & $\mathrm{Ca}^{2+}{ }_{\mathrm{ss}}=-4,9884^{*}+136,0290^{* * *} \mathrm{~K}_{\mathrm{fc}}-0,7913^{*} \mathrm{P}_{\mathrm{fc}}$ & 74,59 \\
\hline Doscientos & $\mathrm{Ca}^{2+}{ }_{s \mathrm{~s}}=-60,6472^{*}+3,4618^{*} \mathrm{CIC}$ & 36,97 \\
\hline Guamal & $\mathrm{Ca}^{2+}{ }_{\mathrm{ss}}=-20,4681^{*}+0,1748 \mathrm{Mn}_{\mathrm{fc}}+1,9400^{* *} \mathrm{~S}_{\mathrm{fc}}$ & 88,11 \\
\hline Quindío & $\mathrm{Ca}^{2+}{ }_{\mathrm{ss}}=36,2045^{* *}+40,8509^{* * *} \mathrm{Mg}_{\mathrm{fc}}-1,6369^{*} \mathrm{Ar}$ & 65,02 \\
\hline Catarina & $\mathrm{Mg}^{2+}{ }_{\mathrm{ss}}=0,0861094+2,19993^{* *} \mathrm{Mg}_{\mathrm{fc}}$ & 47,74 \\
\hline Chinchiná & $\mathrm{Mg}_{\mathrm{ss}}^{2+}=22,4601^{* *}-4,48932 * \mathrm{pH}_{\mathrm{fc}}+6,5312^{* *} \mathrm{Mg}_{\mathrm{fc}}$ & 67,75 \\
\hline Doscientos & $\mathrm{Mg}^{2+}{ }_{\mathrm{ss}}=3,41542+1,7292^{*} \mathrm{Zn}_{\mathrm{fc}}$ & 32,23 \\
\hline Guamal & $\begin{array}{l}\mathrm{Mg}^{2+}{ }_{\mathrm{ss}}=-120,0^{* * *}+20,8989^{* * *} \mathrm{pH}_{\mathrm{fc}}+0,05369^{*} \mathrm{Mn}_{\mathrm{fc}}-1,59292^{*} \mathrm{Zn}_{\mathrm{fc}}+0,856086^{* *} \mathrm{~S}_{\mathrm{fc}}+ \\
17,2931^{*} \mathrm{~K}_{\mathrm{fc}}\end{array}$ & 97,30 \\
\hline Quindío & $\mathrm{Mg}_{\mathrm{ss}}^{2+}=7,65861^{*}+11,1286^{* * *} \mathrm{Mg}_{\mathrm{fc}}-0,366762^{*} \mathrm{Ar}$ & 62,50 \\
\hline Catarina & $\mathrm{K}^{+}{ }_{\mathrm{ss}}=-2,0681+33,9563^{* * *} \mathrm{~K}_{\mathrm{fc}}$ & 94,68 \\
\hline Chinchiná & $\mathrm{K}^{+}{ }_{\mathrm{ss}}=24,1982^{* *}-4,4647^{* *} \mathrm{pH}_{\mathrm{fc}}+24,5280^{* * *} \mathrm{~K}_{\mathrm{fc}}-0,1535^{* *} \mathrm{Ar}$ & 79,77 \\
\hline Doscientos & $\mathrm{K}^{+}{ }_{\mathrm{ss}}=-3,9774^{*}+21,5759^{* * *} \mathrm{~K}_{\mathrm{fc}}$ & 89,08 \\
\hline Guamal & $\mathrm{K}^{+}{ }_{\mathrm{ss}}=-2,5889^{*}+21,0159^{* * *} \mathrm{~K}_{\mathrm{fc}}$ & 79,06 \\
\hline Quindío & $\mathrm{K}^{+}{ }_{\mathrm{ss}}=17,6365+0,6972^{* * *} \mathrm{Mn}_{\mathrm{fc}}-1,2054^{*} \mathrm{Ar}$ & 81,70 \\
\hline
\end{tabular}

ss $=$ solución del suelo; ${ }_{f c}=$ fase de cambio; ${ }^{*}=$ valor $p<0,05 \geq 0,01 ;{ }^{* *}=$ valor $p<0,01 \geq 0,001 ;{ }^{* *}=$ valor $p<0,001$

\section{Conclusiones}

Los resultados obtenidos permiten concluir que:

Las concentraciones de $\mathrm{Ca}^{2+} \mathrm{Mg}^{2+} \mathrm{y} \mathrm{K}^{+}$en la fase de cambio y la solución del suelo no presentaron diferencia estadística en los primeros $30 \mathrm{~cm}$ del perfil; salvo algunas excepciones, donde la concentración fue mayor en los primeros $5 \mathrm{~cm}$ de profundidad, posiblemente debido a la actividad orgánica en la rizosfera.

Las concentraciones de los cationes objeto de estudio fueron estadísticamente diferentes entre unidades; de las cuales las unidades provenientes de materiales de composición ultramáfica y máfica exhibieron los mayores contenidos de $\mathrm{Ca}^{2+} \mathrm{Mg}^{2+}$ y $\mathrm{K}^{+}$, mientras que los provenientes de materiales intermedios presentaron los menores contenidos. Lo anterior se explica porque los materiales máficos tienen mayores contenidos de piroxenos, anfíboles y plagioclasas, los cuales son la mayor fuente primaria de $\mathrm{Ca}^{2+} \mathrm{Mg}^{2+}$ y $\mathrm{K}^{+}$en las reservas del suelo.

Los coeficientes de variación para las bases de interés fueron de medios a altos, lo cual es atribuido a la naturaleza de los materiales y al manejo agronómico de los lotes; lo cual ratifica la importancia de contemplar las fuentes de variación naturales y antrópicas del suelo, como factor determinante del diseño de muestreo.

El catión predominante, en la totalidad de las unidades estudiadas y para ambas fases, fue $\mathrm{Ca} 2+$, seguido por $\mathrm{Mg}^{2+}$ y $\mathrm{K}^{+}$. La menor concentración 
en $\mathrm{K}+$ se puede relacionar con su monovalencia, la de $\mathrm{Mg}^{2+}$ se atribuye a su mayor al radio de hidratación, el cual es retenido con menos fuerza que el $\mathrm{Ca}^{2+;}$ y este último, por tener menor radio de hidratación es retenido con mayor fuerza por los coloides del suelo y es menos complejado, lixiviado y/o precipitado, quedando en mayor concentración para las plantas, en comparación con los otros dos cationes.

Las unidades Guamal, Doscientos y Catarina poseen una fertilidad potencial mayor que las unidades Chinchiná y Quindío, sin embargo, las cinco son de fertilidad potencial alta, debido a que poseen una reserva de minerales de fácil meteorización, los cuales al degradarse aportan elementos básicos para el crecimiento vegetal, además de formar minerales de arcillas.

Las concentraciones de $\mathrm{Ca} 2+, \mathrm{Mg} 2+$ y $\mathrm{K}+$ en la solución del suelo, se explicaron por la fase de cambio mediante modelos lineales que incluyeron nutrientes del suelo y propiedades como $\mathrm{CIC}, \mathrm{pH}$ y contenido de arcillas. Las concentraciones de Ca2+ en la solución se explicaron desde 36,97\% hasta $88,11 \%$ por la fase de cambio, las de Mg2+ desde $32,23 \%$ hasta $97,30 \%$ y las de $\mathrm{K}+$ desde $79,06 \%$ hasta $94,68 \%$.

\section{Agradecimientos}

Los autores presentan sus agradecimientos a la ONG Solidaridad Regional Andes, como cofinanciadora de la investigación, especialmente al Ing. Carlos Isaza; a la Universidad de Manizales - Maestría en Desarrollo Sostenible y Medio Ambiente, a Jhon Fredy Betancur; a los colaboradores de la disciplinas de suelos y biometría de Cenicafé: Alveiro Salamanca, Andrés Felipe Castro, Arturo Gómez, Esther Cecilia Montoya, Norbey Alzate, Rubén Medina, Vanessa Catalina Díaz y Wadi Andrey Castaño, y al Comité Departamental de Cafeteros de Risaralda, principalmente a los ingenieros Daniel Mauricio Gómez, Juan Carlos Gómez, Norberto Rincón y Sandra Milena García.

\section{Literatura citada}

1. Anda, M. \& Sarwani, M. (2012). Mineralogy, chemical composition, and dissolution of fresh ash eruption: new potential source of nutrients. Soil Sci. Soc. Am. J. 76, 733-747.

2. Anda, M., Suryani, E. \& Subardja, D. (2015). Strategy to reduce fertilizer application in volcanic paddy soils: Nutrient reserves approach from parent materials. Soil and Tillage Research, 150, 10-20.

3. Arias, E., Sadeghian, S., Mejía, B. \& Morales, C.S. (2009). Lixiviación del nitrógeno en algunos suelos de la zona cafetera y su relación con la textura. Cenicafé 60 (3): 239-252.

4. Cambardella, C.A. (1994). Field scale variability of soil properties in central lowa. Soil Sci Soc AM J 58(5): 1501-1511.

5. Chen, G., Weil, R. \& Hill, R.L. (2014). Effects of compaction and cover crops on soil least limiting water range and air permeability. Soil and Tillage Research, 136, 61-69.

6. Emamgolizadeh, S., Bateni, S.M., Shahsavani, D., Ashrafi, T. \& Ghorbani, H. (2015). Estimation of soil cation exchange capacity using Genetic Expression Programming (GEP) and Multivariate Adaptive Regression Splines (MARS). J. Hydrol., http://dx.doi.org/10.1016/j. jhydrol.2015.08.025.

7. Henao, M.C. (1995). Evaluación de la disponibilidad del magnesio en dos suelos de la zona cafetera mediante índices relacionados con los factores cantidad e intensidad. Revista Suelos Ecuatoriales 25, 47-50.

8. Henao, M.C. \& Delvaux, B. (2000). Lixiviación de elementos fertilizantes en algunos andisoles de la zona cafetera central colombiana. X Congreso Colombiano de la Ciencia del Suelo Ponencia. Colombia.

9. Henao, M.C. \& Hernández, E. (2002). Disponibilidad de potasio en suelos derivados de cenizas volcánicas y su relación con la nutrición del café en la etapa vegetativa. CENICAFE, 53(4), 293-305.

10. Hincapie, E. \& Henao, M.C. (2008). Efecto de la fertilización del café sobre la composición de la fase líquida en suelos derivados de cenizas volcánicas. Congreso Colombiano de la Ciencia del Suelo, 14. 29-31. Villavicencio (Colombia): capitulos de memorias.

11. Kim, J.Y. \& Kim, Y. (2015). Sorption of cesium on weathered biotite: The effects of cations in solution. $C A-$ TENA, 135, 107-113.

12. Leao, T.P., Da Silva, A.P., Macedo, M.C.M., Imhoff, S. \& Euclides, V.P.B. (2006). Least limiting water range: A potential indicator of changes in near-surface soil physical quality after the conversion of Brazilian Savanna into pasture. Soil and Tillage Research, 88(1), 279-285.

13. Lindsay, W.L. (1979). Chemical equilibria in soil. New York: John Wiley and Sons.

14. Lince, L.A. \& Sadeghian, S. (2012). Número de muestras simples para el análisis de las propiedades del suelo. Suelos ecuatoriales 42(2):129-137. 2012 
15. Maathuis, F.J. (2009). Physiological functions of mineral macronutrients. Current Opinion in Plant Biology, 12(3), 250-258.

16. Melo, V.D.F. \& Alleoni, L.R.F. (2009). Química e mineralogia do solo. Parte I, Viçosa-MG, 529.

17. Sadeghian, S. (2010). Evaluación de la fertilidad del suelo para una adecuada nutrición de los cultivos. Caso café. XII Congreso ecuatoriano de la Ciencia del Suelo, 23. Santo Domingo.

18. Sadeghian, S., Mejía, M.B. \& Arcila, P.J. (2006). Composición elemental de frutos de café y extracción de nutrientes para la cosecha en la zona cafetera de Colombia. CENICAFÉ 57(4):251-261.

19. Sadeghian, S. \& Zapata, R. (2012). Propiedades relacionadas con la adsorción de cationes intercambiables en algunos suelos de la zona cafetera colombiana. $C E-$ NICAFÉ 63 (2):79-89.

20. Sadeghian, S. (2012). Efecto de los cambios en las relaciones de calcio, magnesio y potasio intercambiables en suelos de la zona cafetera colombiana sobre la nutrición de café (Coffea arabica L.) en la etapa de almácigo. Medellín, Colombia: Universidad Nacional de Colombia - Facultad de Ciencias Agropecuarias - Doctorado en Ciencias Agropecuarias.

21. Sadeghian, S. (2014). Manejo integrado de nutrientes para una caficultura sostenible. Suelos Ecuatoriales 44(2): 74-89.
22. Safadoust, A., Feizee, P., Mahboubi, A.A., Gharabaghi, B., Mosaddeghi, M.R. \& Ahrens, B. (2014). Least limiting water range as affected by soil texture and cropping system. Agricultural Water Management, 136, 34-41.

23. Smethurst, P.J. (2000). Soil solution and other soil analyses as indicators of nutrient supply: a review. Forest Ecology and Management, 138(1), 397-411.

24. Sparks, D.L. (2003). Environmental soil chemistry. San Diego. Academic Press 352 p.

25. Tormena, C.A., da Silva, A.P. \& Libardi, P.L. (1999). Soil physical quality of a Brazilian Oxisol under two tillage systems using the least limiting water range approach. Soil and Tillage Research, 52(3), 223-232.

26. Wilson, M.G., Sasal, M.C. \& Caviglia, O.P. (2013). Critical bulk density for a Mollisol and a Vertisol using least limiting water range: Effect on early wheat growth. Geoderma, 192, 354-361.

\section{Conflicto de Intereses}

Los autores declaran no tener ningún conflicto de intereses 